\title{
TwiSe at SemEval-2017 Task 4: Five-point Twitter Sentiment Classification and Quantification
}

\author{
Georgios Balikas \\ University of Grenoble Alps, CNRS, Grenoble INP - LIG \\ georgios.balikas@imag.fr
}

\begin{abstract}
The paper describes the participation of the team "TwiSE" in the SemEval-2017 challenge. Specifically, I participated at Task 4 entitled "Sentiment Analysis in Twitter" for which I implemented systems for five-point tweet classification (Subtask C) and five-point tweet quantification (Subtask E) for English tweets. In the feature extraction steps the systems rely on the vector space model, morpho-syntactic analysis of the tweets and several sentiment lexicons. The classification step of Subtask C uses a Logistic Regression trained with the one-versus-rest approach. Another instance of Logistic Regression combined with the classify-and-count approach is trained for the quantification task of Subtask E. In the official leaderboard the system is ranked 5/15 in Subtask C and 2/12 in Subtask E.
\end{abstract}

\section{Introduction}

Microblogging platforms like Twitter have lately become ubiquitous, democratizing the way people publish and access information. This vast amount of information that reflects the opinions, news or comments of people creates several opportunities for opinion mining. Among other platforms, Twitter is particularly popular for research due to its scale, representativeness and ease of access to the data it provides. Furthermore, to facilitate the study of opinion mining, high quality resources and data challenges are organized. The Task 4 of the SemEval-2017 challenges, entitled "Sentiment Analysis in Twitter" is among them.

The paper describes the participation of the team Twitter Sentiment (TwiSe) in two of the subtasks of Task 4 of SemEval-2017. Specifically,
I participated in Subtasks C and E. Both of them assume that sentiment is distributed across a fivepoint scale ranging from VeryNegative to VeryPositive. Subtask C is a sentiment classification task, where given a tweet the aim is to assign one of the five classes. Subtask E is a quantification task, whose aim is given a set of tweets referring to a subject to estimate the prevalence of each of the five classes. The tasks are described in more detail at (Rosenthal et al., 2017).

The rest of the paper is organized as follows: Section 2 describes the feature extraction steps performed in order to construct the representation of a tweet, which is the same for both subtasks C and E. Section 3 details the learning approaches used and Section 4 summarizes the achieved performance. Finally, Section 5 concludes with pointers for future work.

\section{Feature Extraction}

In this section I describe the details of the feature extraction process performed. My approach is heavily inspired by my previous participation in the "Twitter Sentiment Analysis" task of SemEval-2016, which is detailed at Balikas and Amini (2016). Importantly, the code for performing the feature extraction steps described below is publicly available at https://github. com/balikasg/SemEval2016-Twitter_ Sentiment_Evaluation.

There are three sets of features extracted:

1. Word occurrence features,

2. Morpho-syntactic features like counts of punctuation and part-of-speech (POS) tags,

3. Semantic features based on sentiment lexicons and word embeddings. 
For the data pre-processing, cleaning and tokenization ${ }^{1}$ as well as for most of the learning steps, I used Python's Scikit-Learn (Pedregosa et al., 2011) and NLTK (Bird et al., 2009).

\subsection{Word occurrence and morpho-syntactic features}

Following (Kiritchenko et al., 2014; Balikas and Amini, 2016) I extract features based on the words that occur in a tweet. The aim is to describe the lexical content of the tweets as well as to capture part of the words order. The latter is achieved using $N$-grams, with $N>1$. To reduce the dimensionality of the representations when using $N$ grams, especially with noisy data such as tweets, I use the hashing trick. Hashing is a fast and space-efficient way for vectorizing text spans. It turns arbitrary features into vector indices of predefined size (Weinberger et al., 2009). For example, assume that after the vocabulary extraction step one has a vocabulary of dimensionality $50 \mathrm{~K}$. This would result in a very sparse vector space model and longer training for a classifier. Feature hashing can be seen as a dimensionality reduction process where a hash function given a textual input (vocabulary item) associates it to a number $j$ within $0 \leq j \leq D$, where $D$ is the dimension of the new representation.

The word-occurrence and morpho-syntactic features I extracted are:

- $N$-grams with $N \in[1,4]$, projected to $20 \mathrm{~K}$ dimensional space using the hashing function, ${ }^{2}$

- character $m$-grams of dimension $m \in[4,5]$, that is sequences of characters of length 4 or 5 , projected to $25 \mathrm{~K}$-dimensional space using the same hashing function. The sizes of the output of the hashing function for $N$-grams and character $m$-grams $(20 \mathrm{~K}$ and $25 \mathrm{~K}$ respectively) were decided using the validation set. Also, I applied the hashing trick only for these two types of features,

- \# of exclamation marks, \# of question marks, sum of exclamation and question marks, bi-

\footnotetext{
${ }^{1}$ We adapted the tokenizer provided at http: //sentiment.christopherpotts.net/ tokenizing.html

${ }^{2}$ I used the signed 32-bit version of Murmurhash3 function, implemented as part of the HashingVectorizer class of scikit-learn.
}

nary feature indicating if the last character of the tweet is a question or exclamation mark,

- \# of capitalized words (e.g., GREAT) and \# of elongated words (e.g. coool), \# of hashtags in a tweet,

- \# of negative contexts. Negation is important as it can alter the meaning of a phrase. For instance, the meaning of the positive word "great" is altered if the word follows a negative word e.g. "not great". We have used a list of negative words (like "not") to detect negation. We assumed that words after a negative word occur in a negative context, that finishes at the end of the tweet unless a punctuation symbol occurs before. Notice that negation also affects the $N$-gram features by transforming a word $w$ in a negated context to $w_{-} N E G$,

- \# of positive emoticons, \# of negative emoticons and a binary feature indicating if emoticons exist in a given tweet, and

- The distribution of part-of-speech (POS) tags (Gimpel et al., 2011) with respect to positive and negative contexts, that is how many verbs, adverbs etc., appear in a positive and in a negative context in a given tweet.

\subsection{Semantic Features}

With regard to the sentiment lexicons, I used:

- manual sentiment lexicons: the Bing liu's lexicon (Hu and Liu, 2004), the NRC emotion lexicon (Mohammad and Turney, 2010), and the MPQA lexicon (Wilson et al., 2005),

- \# of words in positive and negative context belonging to the word clusters provided by the CMU Twitter NLP tool ${ }^{3}$, \# of words belonging to clusters obtained using skip-gram word embeddings,

- positional sentiment lexicons: the sentiment 140 lexicon (Go et al., 2009) and the Hashtag Sentiment Lexicon (Kiritchenko et al., 2014)

I make, here, more explicit the way I used the sentiment lexicons, using the Bing Liu's lexicon as an example. I treated the rest of the lexicons similarly, which is inspired by (Kiritchenko et al.,

\footnotetext{
${ }^{3}$ http: //www.cs.cmu.edu/ ark/TweetNLP/
} 
2014). For each tweet, using the Bing Liu's lexicon I generated a 104-dimensional vector. After tokenizing the tweet, I count how many words (i) in positive/negative contexts belong to the positive/negative lexicons (4 features) and I repeat the process for the hashtags (4 features). To this point one has 8 features. I repeat the generation process of those 8 features for the lowercase words and the uppercase words. Finally, for each of the 24 POS tags the (Gimpel et al., 2011) tagger generates, I count how many words in positive/negative contexts belong to the positive/negative lexicon. As a result, this generates $2 \times 8+24 \times 4=104$ features in total for each tweet based on the sentiment lexicons.

With respect to the features from text embeddings, I opt for cluster-based embeddings inspired by (Partalas et al., 2016). I used an in-house collection of $\sim 40 \mathrm{M}$ tweets collected using the Twitter API between October and November 2016. Using the skip-gram model as implemented in the word2vec tool (Mikolov et al., 2013), I generated word embeddings for each word that appeared in the collected data more than 5 times. Therefore, each word is associated with a vector of dimension $D$, where I set $D=100$, which I did not validate. Then, using the k-means algorithm I clustered the learned embeddings, initializing the clusters centroids with k-means++ (Arthur and Vassilvitskii, 2007). Having the result of the clustering step, I produced binary cluster membership features for the words of a tweet. For instance, assuming access to the results of k-means with $k=50$, each tweet's representation is augmented with 50 features, denoting whether words of the tweet belong to each of the 50 clusters. The number of the clusters $k$ in the $\mathrm{k}$-meams algorithm is a hyperparameter, which was set to 1,000 after tuning it from $k \in\{100,250,500,1000,1500,2000\}$.

\section{The Learning Approach}

The section describes the learning approach for Subtasks C and E. For each of them, I used a Logistic Regression optimized with the Broyden-Fletcher-Goldfarb-Shanno (BFGS) algorithm from the quasi-newton family of methods, and in particular its limited-memory (L-BFGS) approximation (Byrd et al., 1995). ${ }^{4}$

\footnotetext{
${ }^{4}$ From scikit-learn: 'LogisticRegression(solver='lbfgs').
}

\subsection{Fine-grained tweet classification}

The output of the concatenation of the representation learning steps described at Section 2 is a 46,368-dimensional vector, out of which $N$-grams and character $m$-grams correspond to $45 \mathrm{~K}$ elements. We normalize each instance using $l_{2}$ norm and this corresponds to the vector representation of the tweets. I train a Logistic Regression as implemented in Scikit-learn (Pedregosa et al., 2011) using $L_{2}$ regularization. The hyper-parameter $C$ that controls the importance of the regularization term in the optimization problem is selected with grid-search from $C \in\left\{10^{-4}, 10^{-3}, \ldots, 10^{4}\right\}$. For grid-search I used a simple train-validation split, which is described in the next section. Once the $C$ parameter is selected, I retrained the Logistic Regression in the union of the instances of the training and validation sets.

In addition, as shown in Figure 1 ("Class Distribution: Training data"), the classification problem is unbalanced as the distribution of the examples across the five sentiment categories is not uniform. To account for this, I assigned class weights to the examples when training the Logistic Regression. The goal is to penalize more misclassification errors in the less frequent classes. The weights are inversely proportional to the number of instances of each class. ${ }^{5}$ This is also motivated by the fact that the official evaluation measure is the macroaveraged Mean Absolute Error $\left(\mathrm{MAE}_{\mathrm{M}}\right.$ ) that is averaged across the different classes and accounts for the distance between the true and predicted class. More information about the evaluation metrics used can be found at (Rosenthal et al., 2017).

\subsection{Fine-grained tweet quantification}

While the aim of classification is to assign a category to each tweet, the aim of quantification is to estimate the prevalence of a category to a set of tweets. Several methods for quantification have been proposed: I cite for instance the work of G. Forman on classify and count and probabilistic classify and count (Forman, 2008) and the recently proposed ordinal quantification trees (Da San Martino et al., 2016). In this work, I focus on a classify and count approach, which simply requires classifying the tweets and then aggregating the classification results. The official evaluation measure is Earth Movers Distance (EMD) averaged over the

\footnotetext{
${ }^{5}$ From scikit-learn: 'LogisticRegression(class_weight $=$ 'balanced').
} 


\begin{tabular}{lc}
\hline & Subtask C \& E \\
\hline Train2016 & 5,482 \\
Development2016 & 1,810 \\
DevTest2016 & 1,778 \\
Test2016 & 20,632 \\
Test2017 & 12,137 \\
\hline
\end{tabular}

Table 1: Size of the data used for training and development purposes. We only relied on the SemEval 2016 datasets.

\begin{tabular}{lclll}
\hline \multicolumn{2}{c}{ Subtask C } & & \multicolumn{2}{c}{ Subtask E } \\
Team & Score & & Team & Score \\
\cline { 1 - 2 } \cline { 5 - 6 } BB_twtr & 0.4811 & & BB_twtr & 0.245 \\
DataStories & 0.5552 & & TwiSe & 0.269 \\
Amobee-C-137 & 0.5993 & & funSentiment & 0.273 \\
Tweester & 0.6234 & & ELiRF-UPV & 0.306 \\
TwiSe & 0.6400 & & NRU-HSE & 0.317 \\
\hline
\end{tabular}

Table 2: Top-5 systems ranks for Subtask C based on $\mathrm{MAE}_{\mathrm{M}}$ and of Subtask E based on EMD.

subjects of the data, described in detail at (Rosenthal et al., 2017).

The classification and the quantification methods I use rely on efficient operations in terms of memory (hashing) and computational resources (linear models). The feature extraction and learning operations are naturally parallellizable. I believe that this is an important advantage, as the end-to-end system is robust and fast to train.

\section{The Experimental Framework}

The data Table 1 shows the data released by the task organizers. To tune the hyper-parameters of my models, I used a simple validation mechanism: I concatenated the "Train2016", "Development2016", and "DevTest2016" (9,070 tweets totally) to use them as training and I left the "Test2016" as validation data. I acknowledge that using the "Test2016" part of the data only for validation purposes may be limiting in terms of the achieved performance, since these data could have also used to train the system. I also highlight that by using more elaborate validation strategies like using the subjects of the tweets, one should be able to achieve better results for tuning.

Official Rankings Table 2 shows the performance the systems achieved. There are two main observations. For Subtask C, where TwiSe is ranked $5^{\text {th }}$, I note that the system is a slightly improved version of the system of (Balikas and Amini, 2016), ranked first in the Subtask in the 2016 edition. The only difference is the addition

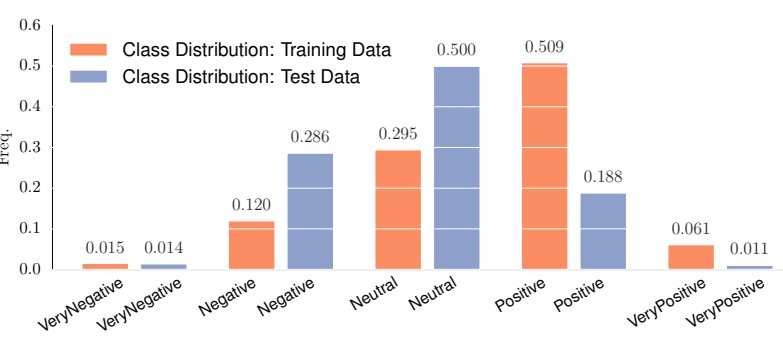

Figure 1: The distribution of the instances in the training and test sets among the five sentiment classes. The figure is rendered better with color.

of the extra features from clustering the word embeddings. This entails that significant progress was made to the task, which is either due to the extra data ("Test2016" we only used for validation) or more efficient algorithms. On the other hand, TwiSe is ranked $2^{\text {nd }}$ in Subtask E. This, along with the simplicity of the approach used that is based on aggregating the counts of the classification step, entails that there is more work to be done in this direction.

Five-Scale Classification: Error Analysis Analyzing the classification errors, one finds out that the (macro-averaged) mean-absolute-error per sentiment category is distributed as follows: VeryNegative: 0.836, Negative: 0.566, Neutral: 0.584 , Positive: 0.771 , VeryPositive: 0.443 . The system performed the best in the VeryPositive class (lowest error) and the worst in the VeryNegative class. Interestingly, the system did not do as well in the Positive class. To better understand why, Figure 1 plots the distribution of the instances across the five sentiment classes, for the training data we used and the test data. Notice how the Positive class is the dominant in the training data, while this changes in the test data. I believe that that the distribution drift, between the training and test data is indicative as of why the system performed poorly in the "Positive" class.

Five-Scale Quantification: Error Analysis I repeat, here, the error analysis process for the quantification task. The best performance was achieved in the subject "leonard cohen", whose EMD was 0.029 while the worst performance in the topics "maduro" (EMD=0.709) and "medicaid" (EMD=0.660). The distribution of sentiment for "leonard cohen" is very similar to the distribution of sentiment in the training set, KullbackLeibner divergence of 0.140 . On the other hand the Kullback-Leibner divergence for "maduro" 
and "medicaid", which are both skewed towards the negative sentiment, are 1.328 and 0.896 respectively. Although a more detailed error analysis is required in order to improve the performance of the system, I believe that the distribution drift between the training examples and the examples of a subject plays an important role. This may be further enhanced by the fact I used a classify and count approach which does not account for drifts.

\section{Conclusion}

The paper described the participation of TwiSe in the subtasks $\mathrm{C}$ and $\mathrm{E}$ of of the "Twitter Sentiment Evaluation" Task of SemEval-2017. Importantly, my system was ranked $2^{\text {nd }}$ in Subtask E, "Five-point Sentiment Quantification" using a simple classify and count approach on top of a Logistic Regression. An interesting future work direction towards improving the system aims at better handling distribution drifts between the training and test data.

\section{References}

David Arthur and Sergei Vassilvitskii. 2007. kmeans++: The advantages of careful seeding. In Proceedings of the eighteenth annual ACM-SIAM symposium on Discrete algorithms. Society for Industrial and Applied Mathematics, pages 10271035 .

Georgios Balikas and Massih-Reza Amini. 2016. Twise at semeval-2016 task 4: Twitter sentiment classification. In SemEval@NAACL-HLT 2016, San Diego, CA, USA, June 16-17, 2016. pages 85-91.

Steven Bird, Ewan Klein, and Edward Loper. 2009. Natural Language Processing with Python. O'Reilly Media.

Richard H. Byrd, Peihuang Lu, Jorge Nocedal, and Ciyou Zhu. 1995. A limited memory algorithm for bound constrained optimization. SIAM J. Scientific Computing 16(5):1190-1208.

Giovanni Da San Martino, Wei Gao, and Fabrizio Sebastiani. 2016. Ordinal text quantification. In Proceedings of the 39th International ACM SIGIR conference on Research and Development in Information Retrieval. ACM, pages 937-940.

George Forman. 2008. Quantifying counts and costs via classification. Data Mining and Knowledge Discovery 17(2):164-206.

Kevin Gimpel, Nathan Schneider, Brendan O'Connor, Dipanjan Das, Daniel Mills, Jacob Eisenstein, Michael Heilman, Dani Yogatama, Jeffrey Flanigan, and Noah A Smith. 2011. Part-of-speech tagging for twitter: Annotation, features, and experiments. In Proceedings of the 49th Annual Meeting of the Association for Computational Linguistics: Human Language Technologies: short papers-Volume 2. Association for Computational Linguistics, pages 42 47.

Alec Go, Richa Bhayani, and Lei Huang. 2009. Twitter sentiment classification using distant supervision. CS224N Project Report, Stanford 1:12.

Minqing $\mathrm{Hu}$ and Bing Liu. 2004. Mining and summarizing customer reviews. In Proceedings of the tenth ACM SIGKDD international conference on Knowledge discovery and data mining. ACM, pages 168177.

Svetlana Kiritchenko, Xiaodan Zhu, and Saif M Mohammad. 2014. Sentiment analysis of short informal texts. Journal of Artificial Intelligence Research pages 723-762.

Tomas Mikolov, Kai Chen, Greg Corrado, and Jeffrey Dean. 2013. Efficient estimation of word representations in vector space. CoRR abs/1301.3781.

Saif M Mohammad and Peter D Turney. 2010. Emotions evoked by common words and phrases: Using mechanical turk to create an emotion lexicon. In Proceedings of the NAACL HLT 2010 workshop on computational approaches to analysis and generation of emotion in text. Association for Computational Linguistics, pages 26-34.

Ioannis Partalas, Cédric Lopez, Nadia Derbas, and Ruslan Kalitvianski. 2016. Learning to search for recognizing named entities in twitter. WNUT 2016 page 171 .

F. Pedregosa, G. Varoquaux, A. Gramfort, V. Michel, B. Thirion, O. Grisel, M. Blondel, P. Prettenhofer, R. Weiss, V. Dubourg, J. Vanderplas, A. Passos, D. Cournapeau, M. Brucher, M. Perrot, and E. Duchesnay. 2011. Scikit-learn: Machine learning in Python. Journal of Machine Learning Research 12:2825-2830.

Sara Rosenthal, Noura Farra, and Preslav Nakov. 2017. SemEval-2017 task 4: Sentiment analysis in Twitter. In Proceedings of the 11th International Workshop on Semantic Evaluation. Association for Computational Linguistics, Vancouver, Canada, SemEval '17.

Kilian Weinberger, Anirban Dasgupta, John Langford, Alex Smola, and Josh Attenberg. 2009. Feature hashing for large scale multitask learning. In Proceedings of the 26th Annual International Conference on Machine Learning. ACM, pages 11131120 .

Theresa Wilson, Janyce Wiebe, and Paul Hoffmann. 2005. Recognizing contextual polarity in phraselevel sentiment analysis. In Proceedings of the conference on human language technology and empirical methods in natural language processing. Association for Computational Linguistics, pages 347-354. 